\title{
Busulphan lung
}

\author{
B. E. HEARD ${ }^{1}$ A N D R. A. COOKE
}

From the Department of Morbid Anatomy, Royal Postgraduate Medical School, Du Cane Road, London, W.12

\begin{abstract}
Histological changes attributable to busulphan were found at necropsy in the lungs of six out of 14 cases of chronic granulocytic leukaemia. The alveoli contained persistent fibrinous oedema converting to fibrous tissue, and also atypical cells. Only one out of seven controls with leukaemia showed fibrinous oedema, and atypical cells were absent. In the lungs from rare cases of fatal pulmonary fibrosis due to busulphan, the histological changes were similar but more severe. It is concluded that busulphan commonly causes fibrinous oedema in chronic granulocytic leukaemia and on rare occasions it becomes severe or persistent enough to cause fatal fibrosis of the lungs. The name busulphan lung is suggested for any of these changes. The term 'intra-alveolar fibrosis' should be used instead of 'interstitial fibrosis', as it had been called by most previous authors. Despite these findings we consider that this valuable drug should be discontinued only in those very rare instances when intra-alveolar fibrosis shows signs of becoming disabling.
\end{abstract}

The standard present-day treatment of chronic granulocytic leukaemia is based on the daily administration of about $4 \mathrm{mg}$. of the alkylating agent busulphan (Myleran) (Galton, 1953). The white-cell count is often controlled effectively by the drug for many years.

A number of side-effects have been encountered with the use of this drug. Sterility and hyperpigmentation are seen frequently and are usually overlooked. On rare occasions dangerous sideeffects are encountered, such as pulmonary fibrosis (Oliner, Schwartz, Rubio, and Dameshek, 1961 ; Leake, Smith, and Woodliff, 1963 ; Koss, Melamed, and Mayer, 1965; Harrold, 1966; Smalley and Wall, 1966). The present study is concerned with this complication. Other dangerous side-effects are suppression of the bone marrow and a disorder with certain clinical features of Addison's disease.

In a case of fatal pulmonary fibrosis following treatment with busulphan we found that the alveoli were occupied by organizing fibrin and that the fibrous tissue appeared to be largely intraalveolar rather than interstitial, as had previously been described. We therefore examined a series of patients who had died from chronic granulocytic leukaemia with and without busulphan therapy and found that organizing fibrinous oedema was common after busulphan therapy but not in the other patients. The process was similar

Present addresses: 1 Department of Pathology, University New Buildings, Teviot Place, Edinburgh, 8; 2 General Hospital, Port Moresby, Papua, New Guinea to that which we had observed previously in hexamethonium lung (Heard, 1962) so we have chosen the term 'busulphan lung' as the title of this paper.

\section{MATERIALS AND METHODS}

Histological sections were collected from 21 patients with chronic granulocytic leukaemia. Fourteen patients had been treated with busulphan; seven had not, having died before busulphan was introduced. Paraffin sections were stained with haematoxylin and eosin, phosphotungstic acid haematoxylin, Gordon and Sweet's silver impregnation of reticulin, Weigert's stain for elastic, and van Gieson's stain for collagen.

\section{RESULTS}

Fibrinous intra-alveolar oedema of the lungs, often becoming organized, was found in the sections of six out of 14 cases of chronic granulocytic leukaemia treated with busulphan. This contrasted sharply with a group of earlier cases treated before busulphan had been introduced; fibrinous oedema was present in only one out of seven of these patients.

The earliest histological changes in the lungs consisted of an intra-alveolar accumulation of fibrin mixed with varying numbers of red cells (Fig. 1); sometimes the fibrin was becoming organized by fibroblasts and replaced by reticulin and collagen. The process occurred in respiratory 


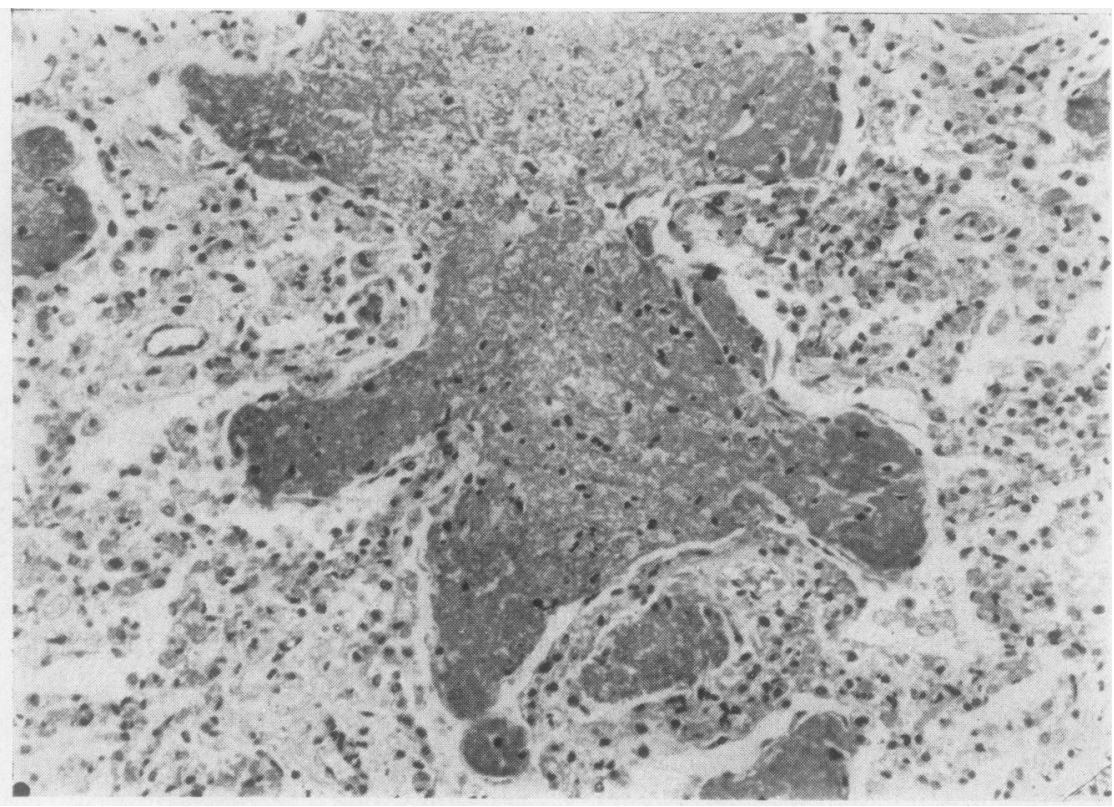

FIG. 1. A coagulum of fibrin and red cells in an alveolar duct of a patient with chronic granulocytic leukaemia treated with busulphan. There are signs of early organization. $($ H.E. $\times 160$.)

bronchioles, alveolar ducts, and alveoli. There were some inflammatory cells among the fibroblasts, including a few eosinophils.

What we believe to be an extreme example of the process just described and diagnosed clinically as pulmonary fibrosis was seen in the lungs of a man of 43 , who received busulphan for two years and developed increasing dyspnoea terminating in death. Chest radiographs showed progressive bilateral diffuse nodular shadowing, and at necropsy (Dr. D. A. Pocock) the lungs were overweight and firm (left 1,160 g., right $1,430 \mathrm{~g}$.) and the external surfaces were finely mottled. The cut surfaces revealed many scattered irregular pale areas, 1 to $2 \mathrm{~mm}$. in diameter. Most parts of the lungs were involved to some extent, but the subpleural region was less affected than the centre, at least inferiorly and posteriorly (Fig. 2). Close examination with the dissecting microscope showed obliteration of many air-spaces in an irregular fashion (Fig. 3). The centres of lobules were not particularly affected, in fact they often seemed to be avoided, and septa were not thickened.

Histologically, the affected parts showed intraalveolar fibrinous oedema in some areas, organizing fibrin in others, and much fibrosis which was predominantly intra-alveolar. Some veins showed intimal fibrosis. The intra-alveolar and $\stackrel{2}{\vec{F}}$ intra-ductal position of the fibrous tissue is parti- $\frac{0}{3}$ cularly well seen with the reticulin stain (Fig. 4), for the centres of the alveolar ducts are sometimeso occupied by long plugs of reticulin-rich tissue. Where the alveolar walls were thickened (inter-으 stitial fibrosis), the appearances supported the possibility that organizing fibrin was incorporatedofrom the lumen into the wall, although this could 3 . not be proved.

In addition to fibrinous oedema and fibrosis, busulphan induced the formation of atypicalo alveolar epithelial cells. These were found in six out of 14 patients receiving busulphan (includingo the patient with severe fibrosis) and in none of the seven patients treated before busulphan was. introduced. They occurred singly and were scat- $N$ tered alike through fibrous and non-fibrous areas. N They were oval or elongated in outline and the largest measured up to $20 \times 40 \mu$, though the average was smaller than that. The plentiful cytoplasm was $\frac{\bar{\Phi}}{\mathrm{C}}$ faintly eosinophilic or basophilic and varied from@ homogeneous to finely granular or even vacuolated in texture (Fig. 5). The nucleus was singleō as a rule, and up to $12 \mu$ across. Its outline was $\stackrel{\odot}{\varnothing}$ round, oval or irregular, and the edge was sharp $\frac{}{\Phi}$ owing to a heavy deposit of peripheral chromatin. Sometimes the whole nucleus was darkly stained, 


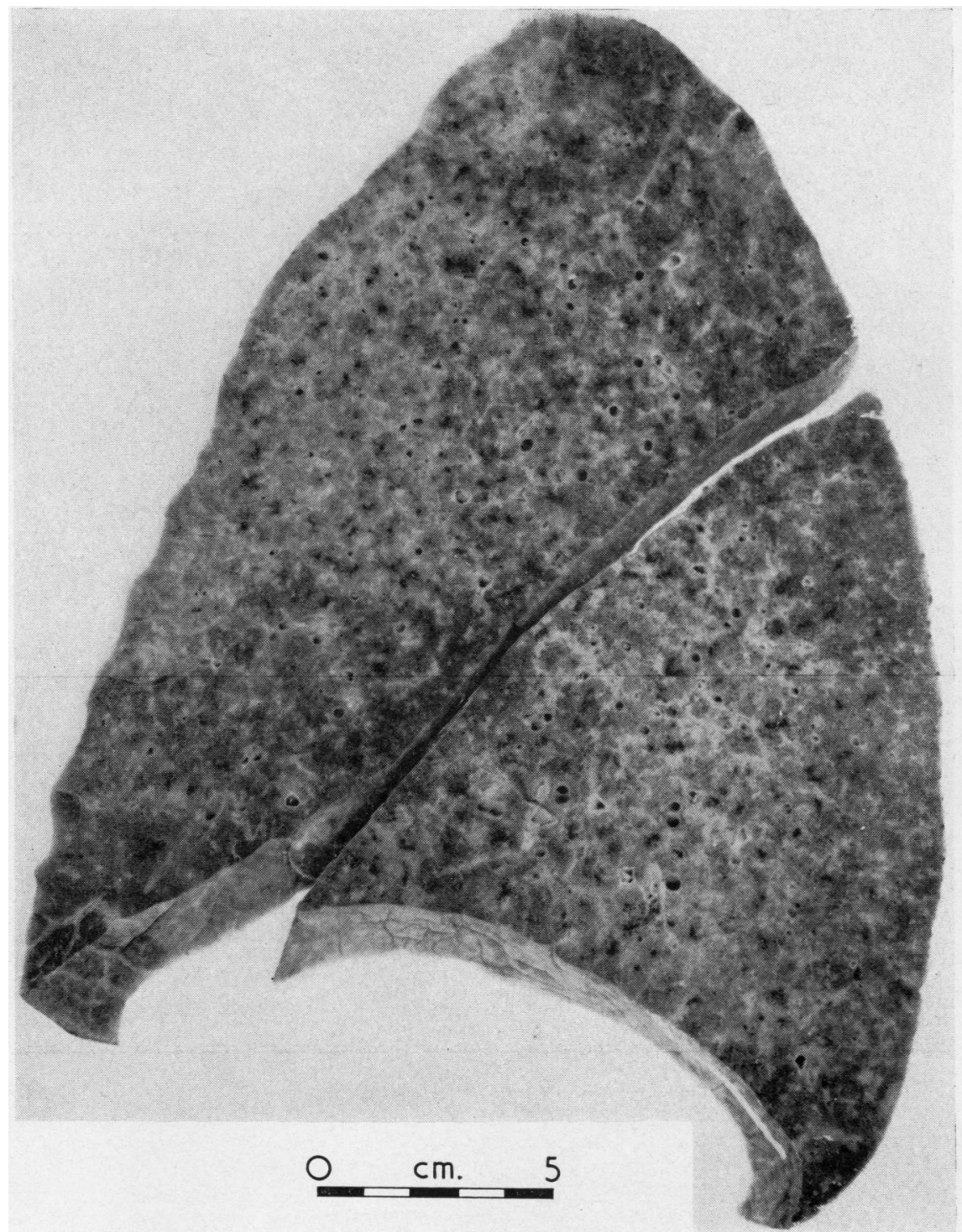

FIG. 2. Fatal pulmonary fibrosis in a man of 43 who received busulphan for two years. Medial aspect of slice of right lung (transverse fissure absent) showing widespread small pale foci of fibrosis affecting the centre more than the subpleural region. See also Figs 3 and 4.

but more often the chromatin was finely stippled in patches or throughout the nucleus, and it was sometimes denser in the centre. Red-staining nucleoli were common and occurred singly or in pairs, and clear nuclear vacuoles of a similar size were sometimes present. Mitoses were absent. There was no resemblance to the changes of cytomegalic inclusion-body disease (Heard, Hassan, and Wilson, 1962).

It was mostly the same cases that showed bizarre cells and organizing fibrin. This will be discussed below, but it should be mentioned here that, although these two features were found in the same lungs, they were not in close association 


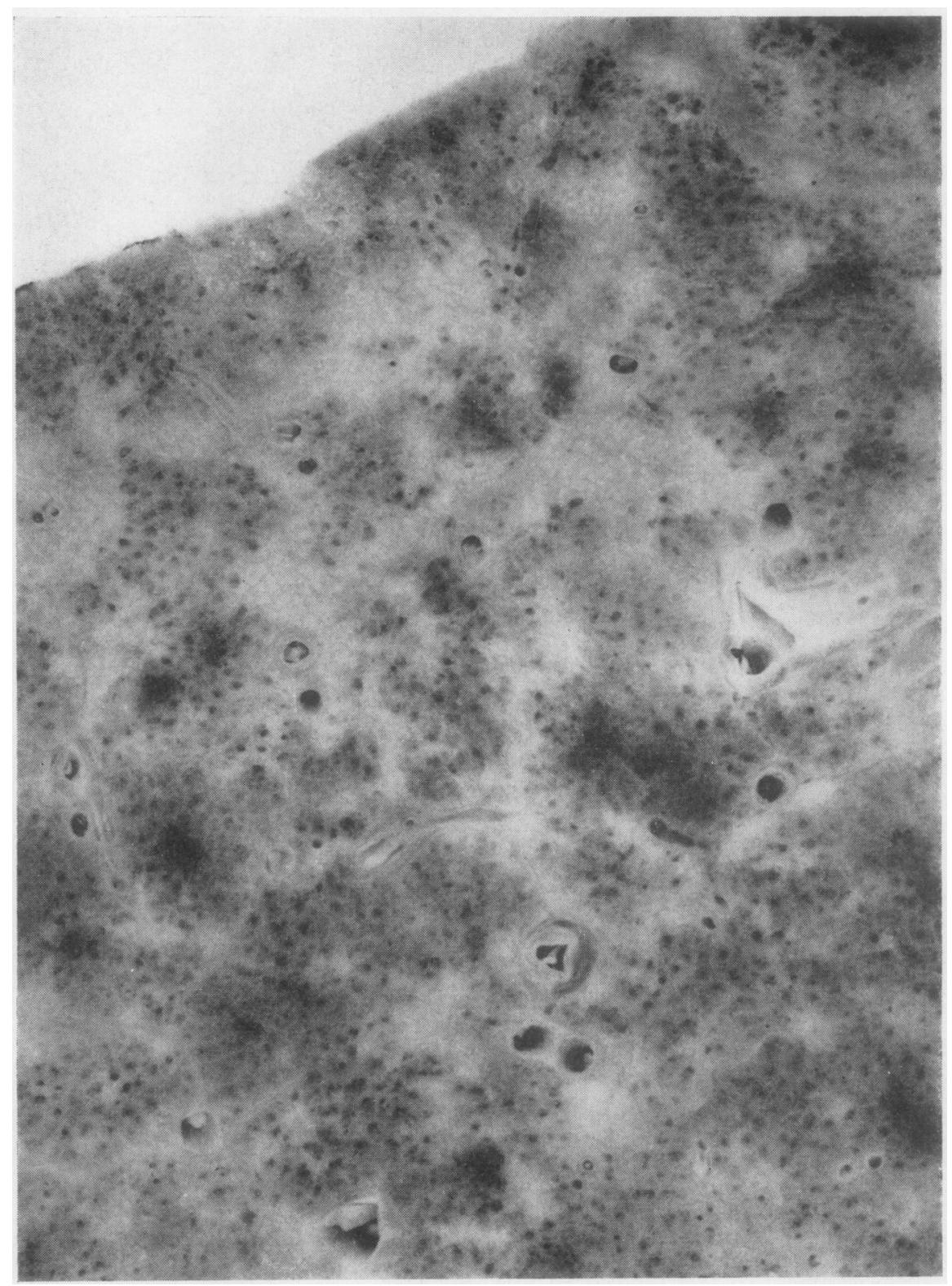

FIG. 3. Higher magnification of anterior segment of upper lobe from Fig. 2 showing irregular fibrosis of fine air-spaces. $(\times 3$.

in particular alveoli. Presumably damaged alveolar walls showed large atypical cells and leaked fibrin. Alternatively, the presence of fibrin stimulated activity in alveolar cells and they reacted abnormally.
DISCUSSION

The frequent occurrence described in this paper of $\frac{O}{\mathbb{Q}}$ fibrinous oedema of the lungs and organization in patients given busulphan for chronic granulo- 


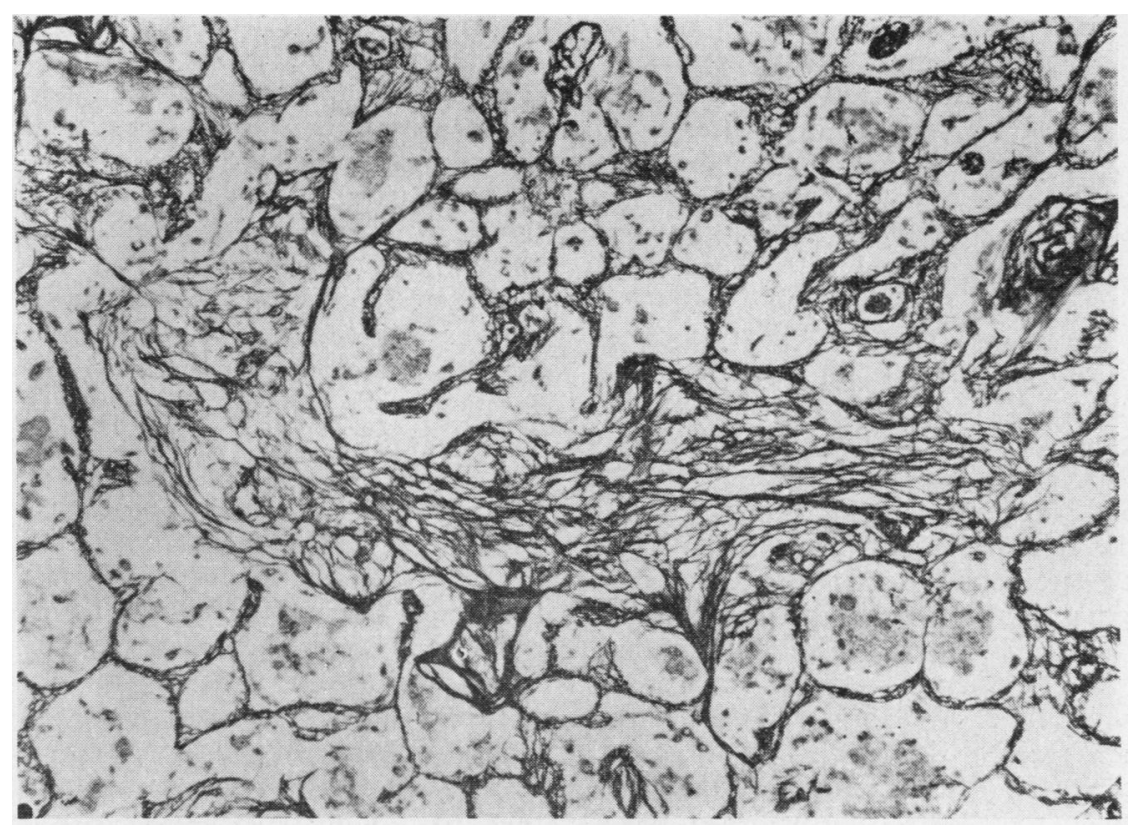

FIG. 4. Histological appearance of lung shown in Figs 2 and 3. The lumen of an alveolar duct is occupied by fibrous tissue. (Reticulin stain. $\times 100$.)

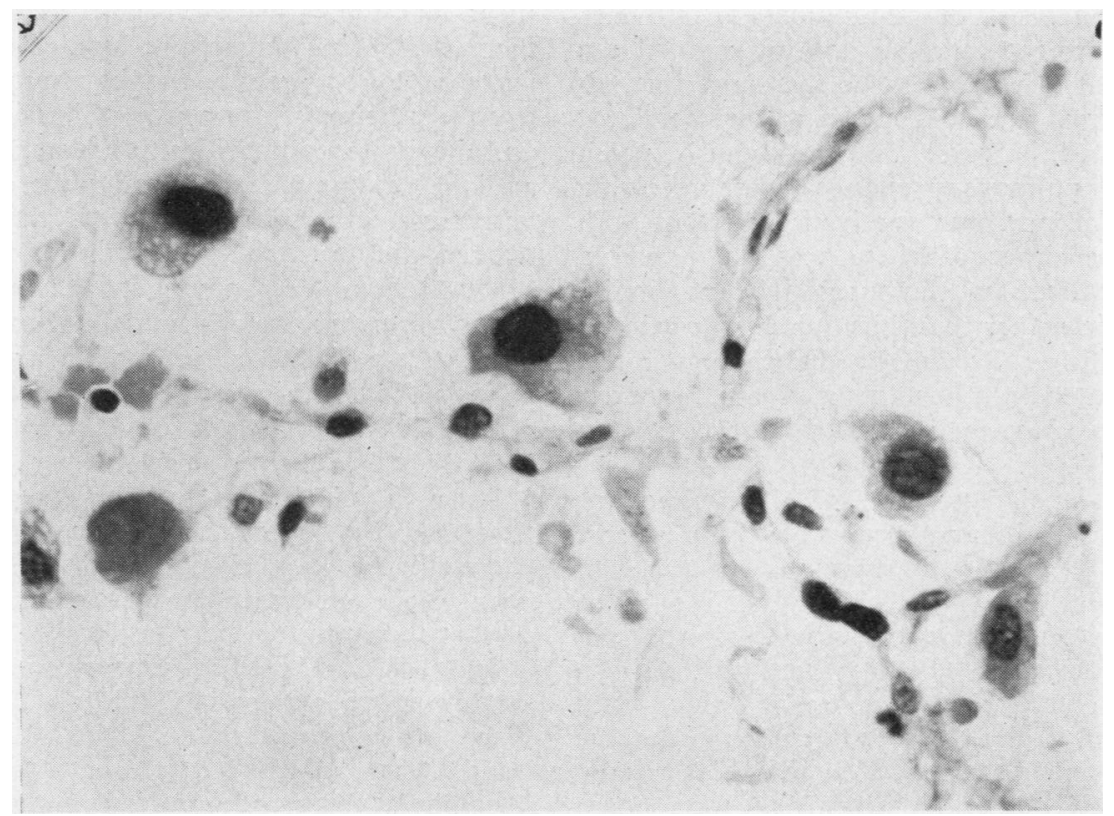

FIG. 5. Large atypical cells in alveoli after busulphan treatment. (H. and E. $\times 520$.) 
cytic leukaemia, and its almost complete absence from similar patients not given busulphan, suggests to us that the drug acts on the alveolar walls in some way to cause the process. The presence of large atypical cells in the alveoli of the same patients supports this. We believe that the same mechanism is responsible for the rarer severe pulmonary fibrosis which is also intraalveolar.

Fibrinous oedema of the lungs is a common finding at necropsy and is usually associated with left ventricular failure. The subject has been reviewed recently by Heard, Steiner, Herdan, and Gleason (1968), who showed changes very similar to the present ones in the lungs of patients with left ventricular failure whether or not uraemia was present. The heart in the present cases showed no consistent gross or microscopical abnormality attributable to either leukaemia or busulphan. The only reference to support a cardiac abnormality is that of Harrold (1966), who described in a patient with pulmonary fibrosis a considerable slowing in atrioventricular conduction time which returned to normal after busulphan therapy was stopped. Other causes of fibrinous pulmonary oedema are inflammation, as in rheumatic fever, $x$-ray treatment involving the lungs, and other drugs. None of the present cases had rheumatic fever. The only infecting agent was Pneumocystis carinii in one case. A careful analysis of the biochemical data and drug treatment revealed nothing apart from busulphan that was likely to be the cause (including blood urea and deep $x$-ray treatment). It should be noted, however, that three out of seven patients with pulmonary changes were receiving mercaptopurine and two received treatment with deep $x$ rays; either of these may have had a contributory effect on the lungs. The possibility that leukaemia rather than busulphan was primarily responsible is ruled out by the findings in the group not treated with busulphan. Again, however, leukaemia may have had a contributory effect on the lungs.

The intra-alveolar position of the pulmonary fibrosis after busulphan therapy has been almost completely disregarded by previous writers who thought it was interstitial. The first report of fibrosis after busulphan therapy was that of Oliner and co-workers (1961), who described in a lung biopsy focal 'chronic pneumonitis' with fibrosis and pleural adhesions, as well as chronic proliferative endarteritis and sometimes thrombi. Scanty lymphocytes and plasma cells were noted and also large atypical alveolar epithelial cells.
Similarly, Leake and co-workers (1963) described $\stackrel{\overrightarrow{\vec{S}}}{\vec{P}}$ 'interstitial fibrosis' in needle biopsies of the lungs 0 of two cases as well as at necropsy. These latter authors kindly lent us sections of their cases, and $\frac{\bar{\rho}}{\frac{\sigma}{\sigma}}$ the appearances are the same as in our case of $\mathbb{Q}$ severe fibrosis, i.e., we think most of the fibrous $\Omega$ tissue is intra-alveolar. Nelson and Andrews s (1964) were more concerned with the large? atypical cells than with the fibrosis. Koss and co- $\vec{\omega}$ workers (1965) appear to have been the first to $\stackrel{\omega}{\omega}$ include intra-alveolar fibrosis in their descriptions ; $\vec{x}$ in three patients with large atypical cells they described fibrosis as nodular and diffuse, peri- $\omega$ bronchial, inter- and intra-alveolar, and sub- $?$ pleural. No histological appearances of the lungs $\stackrel{\infty}{y}$ were given by Harrold (1966), but Smalley and 0 Wall (1966) described 'interstitial fibrosis' in a lung biopsy and also 'bilateral organizing pneu- $z$ monia with interstitial fibrosis' at necropsy.

None of the previous authors appears to have $\stackrel{\rho}{\mathcal{S}}$ noted the common occurrence of fibrinous oedema $\vec{\theta}$ after busulphan therapy or its probable relation- $\infty$ ship to intra-alveolar fibrosis through organization. We found this process to be as distinct histologically in some patients after busulphan therapy as it was with left ventricular failure. We presume that increased permeability of alveolar $\frac{}{1}$ capillaries or venules is common to both circum- $\varrho$ stances and that it is due in one to the drug acting $\overrightarrow{\overrightarrow{0}}$ on the alveolar wall, possibly through the develop- $\exists$ ment of hypersensitivity, and in the other to increased intravascular pressure. Oliner and coworkers (1961) suggested that hypersensitivity to the drug was important in causing fibrosis of the lung, and they noted similarities to hexamethonium lung and the similar chemical structures of busulphan and hexamethonium. However, they also considered the possibility of a radiomimetic action of busulphan. We can support the resemblance to hexamethonium lesions on the further grounds $\frac{}{\partial}$ that the organizing intra-alveolar fibrin and $?$ fibrosis described in the present article occurs also after treatment with hexamethonium (Doniach, $\mathbb{N}$ Morrison, and Steiner, 1964 ; Heard, 1962).

Large atypical cells in the alveoli have been described by several authors, and this peculiar $\mathrm{\omega}$ change is not confined to the lungs. They have been seen after busulphan therapy in the bronchialo and bronchiolar epithelium (Waller, 1960 ; Koss $\underset{\mathbb{D}}{\mathbb{2}}$ et al., 1965), in uterine cervical smears arousing $\stackrel{?}{+}$ suspicions of malignancy (Güreli, Denham, and $\underline{T}$ Root, 1963 ; Koss et al., 1965), throughout the $\frac{\dot{O}}{\mathbb{D}}$ body of an infant born to a mother receiving $\stackrel{?}{\mathcal{P}}$ busulphan (Diamond, Anderson, and McCreadie, $\stackrel{\odot}{\complement}$ 1960 ), in reticulum cells in the bone marrow, and 
in other cells in lymph nodes, pancreas, thyroid, adrenals, kidneys, liver, and many other sites (Güreli et al., 1963 ; Nelson and Andrews, 1964 ; Ward, Konikov, and Reinhard, 1965 ; Koss et al., 1965 ; Oliner et al., 1961).

In animals, marked polyploidy and reduction in mitoses has occurred after busulphan treatment (Chèvremont, Baeckeland, and Frederic, 1960) but not pulmonary fibrosis, though it may not have been sought particularly. Cataracts have been produced in rats (Solomon, Light, and De Beer, 1955).

Cellular atypia is not confined to busulphantreated leukaemia. It has been reported, for example, in the intestinal tract of children with acute leukaemia treated with steroids, antimetabolites, antibacterial drugs, nitrogen mustard, urethane, and $x$ rays (Weston and Guin, 1955).

The cases with large atypical cells in the alveoli were also usually those with fibrinous intraalveolar oedema and fibrosis. In histological sections the two changes were sometimes but not always associated within the same alveolus. There are several possible explanations for this. We believe we have shown that both phenomena are due to busulphan and we suggest that they may occur together because fibrin in the alveolus stimulates alveolar lining cells to enlarge (as in left ventricular failure) and such stimulated cells, modified by busulphan, may undergo bizarre changes. Alternatively, certain patients may be more sensitive to the drug than others, and in these the lungs may show signs of this susceptibility both by developing atypical cells and by leaking fibrin. The lone character of the atypical cells is in keeping with their being abnormal, large alveolar epithelial cells (type B) rather than the smaller type A cells. Klaus, Reiss, Tooley, Piel, and Clements (1962) suggested that normal large alveolar epithelial cells secreted the alveolar lining substance (surfactant) which normally reduces the surface tension of the alveolar walls. If this is so, an abnormality in them would increase the surface tension, which in turn would increase the 'suction' pressure on the alveolar capillaries and lead to pulmonary oedema. This would explain in yet another way the association of these two phenomena.

Although the evidence reported here indicates that the lungs are affected by busulphan more commonly than was formerly believed, we do not consider the changes sufficient for us to recommend less use of this valuable drug. The pulmonary changes only very rarely appear to cause disablement, and the enormous benefits of the drug in the treatment of chronic granulocytic leukaemia far outweigh the dangers.

We are indebted to Dr. David Galton, who was the first to use busulphan clinically, for his help and interest in this work and for allowing us access to the clinical notes of his cases. A clinicopathological presentation with Drs. Galton and Lipton was given to the British Society for Haematology at St. Bartholomew's Hospital, London, on 25 March 1966. We are also grateful to Drs. Eleanor Leake, W. G. Smith, and $H$. J. Woodliff for allowing us to study sections of their cases of pulmonary fibrosis after busulphan therapy. We wish to thank Professors C. V. Harrison and J. V. Dacie for advice, the technical staff for sections, and Mr. W. Brackenbury for the photographs.

\section{REFERENCES}

Chèvremont, M., Baeckeland, E., and Frederic, J. (1960). Contribution cytochimique et histoautoradiographique à l'étude du dans des cellules animales cultivées in vitro. I. Études cytophotométrique et histoautoradiographique des ADN dans des fibroblastes traité par le Myleran. Biochem. Pharmacol., 4, 57.

Diamond, I., Anderson, M. M., and McCreadie, S. R. (1960). Transplacental transmission of busulfan (Myleran) in a mother with leukemia. Production of fetal malformation and cytomegaly. Pediatrics, 25, 85.

Doniach, I., Morrison, B., and Steiner, R. E. (1954). Lung changes during hexamethonium therapy for hypertension. Brit. Heart J., 16, 101 .

Galton, D. A. G. (1953). Myleran in chronic myeloid leukaemia: results of treatment. Lancet, 1, 208.

Güreli, N., Denham, S. W., and Root, S. W. (1963). Cytologic dysplasia related to busulfan (Myleran) therapy; report of a case. Obstet. and Gynec., 21, 466.

Harrold, B. P. (1966). Syndrome resembling Addison's disease following prolonged treatment with busulphan. Brit. med. J., $1,463$.

Heard, B. E. (1962). Fibrous healing of old iatrogenic pulmonary oedema ('hexamethonium lung'). J. Path. Bact., 83, 159.

- Hassan, A. M., and Wilson, S. M. (1962). Pulmonary cytomegalic inclusion-body disease in a diabetic. J. clin. Path., 15, 17.

Steiner, R. E., Herdan, A., and Gleason, D. (1968). Oedema and fibrosis of the lungs in left ventricular failure. Brit. $J$. Radiol., in press.

Klaus, M., Reiss, O. K., Tooley, W. H., Piel, C., and Clements, J. A. (1962). Alveolar epithelial cell mitochondria as source of the surface-active lung lining. Science, 137, 750.

Koss, L. G., Melamed, M. R., and Mayer, K. (1965). The effect of busulfan on human epithelia. Amer. J. clin. Path., 44, 385.

Leake, E., Smith, W. G., and Woodliff, H. J. (1963). Diffuse interstitial pulmonary fibrosis after busulphan therapy. Lancet, 2,432.

Nelson, B. M., and Andrews, G. A. (1964). Breast cancer and cytologic dysplasia in many organs after busulfan (Myleran). Amer. J. clin. Path., 42, 37.

Oliner, H., Schwartz, R., Rubio, F., and Dameshek, W. (1961). Interstitial pulmonary fibrosis following busulfan therapy. Amer. J. Med., 31, 134.

Smalley, R. V., and Wall, R. L. (1966). Two cases of busulfan toxicity. Ann. intern. Med., 64, 154.

Solomon, C., Light, A. E., and De Beer, E. J. (1955). Cataracts produced in rats by 1.4-dimethanesulfonoxybutane (myleran). Arch. Ophthal. (Chic.), 54, 850.

Waller, U. (1960). Riesenkerne nach Mylerantherapie und Milzbestrahlung bei chronischer myeloischer Leukaemie. Path. et Microbiol. (Basel), 23, 283.

Ward, H. N., Konikov, N., and Reinhard, E. H. (1965). Cytologic dysplasia occurring after busulfan (Myleran) therapy. Ann. intern. Med., 63, 654 .

Weston, J. T., and Guin, G. H. (1955). Epithelial atypias with chemotherapy in 100 acute childhood leukemias. Cancer (Philad.), 8, 179. 